\title{
THE LEGACY OF CAMILLO POSSIO TO UNSTEADY AERODYNAMICS
}

\author{
R. Voss, ${ }^{1}$ \\ ${ }^{1}$ DLR Institute of Aeroelasticity, Goettingen, ralph.voss@dlr.de *
}

\begin{abstract}
First a brief overview is given of Camillo Possio's short but outstanding and fruitful career. This is followed by an outline of the state of the art in flutter and unsteady aerodynamic research, and the challenges and problems like high-speed flight that arose in aircraft development at that time. Possio's first publications on gas dynamic and supersonic problems are reviewed. The main focus is on the 1938 report on unsteady subsonic compressible 2D flow that became famous and was named after him, because he was the first person to developed an unsteady compressible aerodynamic theory, which was urgently needed in those years. The theory, which is based on Prandtl's acceleration potential is briefly outlined. Some discussions and comments that took place in Germany and other countries at that time highlight the importance of this work for the scientific community. Early solutions of Possio's integral equation developed by himself and later ones developed by other researchers are presented, as well as approaches that extended the theory to 3 dimensional flows before the war, like Kuessner's theory, which was probably influenced by Possio. Finally Camillo Possio's later scientific contributions to wind tunnel interference and to hydrodynamics are described. A summary of some developments of the 2 nd half of the 20th century demonstrate that Camillo Possio created a milestone for modern aircraft research during his very short career.
\end{abstract}

keywords: Aeroelasticity, Unsteady Aerodynamics, Flutter, Integral Equation, Possio.

\section{Introduction}

Camillo Possio was born on October 30. 1913 and died on April 5. 1945. He was killed by a bomb during the last air-raid over Turin. He received the Laureate Degree in Industrial Engineering and in Aeronautics, both in Turin in 1936 and 1937. He was a pupil of Modesto Panetti and Carlo Ferrari. He was Assistant Professor and then Professor at Politecnico di Torino. His work is well known in the world's aeronautic community, particularly his method

\footnotetext{
${ }^{*}$ The author would like to thank Prof. H. Foersching for many fruitful discussions.
}

Please use the following format when citing this chapter:

Author(s) [insert Last name, First-name initial(s)], 2006, in IFIP International Federation for Information Processing, Volume 199, System Modeling and Optimization, eds. Ceragioli F., Dontchev A., Furuta H., Marti K., Pandolfi L., (Boston: Springer), pp. [insert page numbers]. 
of calculating the unsteady airloads of harmonically oscillating wings in $2 \mathrm{D}$ compressible flow, the key equation of which has been named after him. But his fruitful scientific carreer also covers general problems of gas dynamics and supersonic flow, 3D wing oscillations in incompressible flow, the influence of free surfaces on hydrodynamics, as well as the influence of wind tunnel walls on measurements. His work was published in about 16 papers, many of which were translated from Italian to German and English. When he died at the age of 31 , he was one of the most promising experts of aerodynamics in Italy. He possessed the special skill of using high-level mathematics to obtain results of practical interest.

\section{Aeroelastic and high-speed flight challenges}

During the fast development of aircraft at the beginning of the last century, the significance of aeroelastic effects soon increased. These phenomena resulted from the interaction of the elastic structural system with a surrounding airflow. Airplanes have to be built extremely lightly and their structure is flexible. Elasticity of an aircraft increases with size more than proportional. Fig. 1depicts the wing deformations of modern aircraft wings in flight.
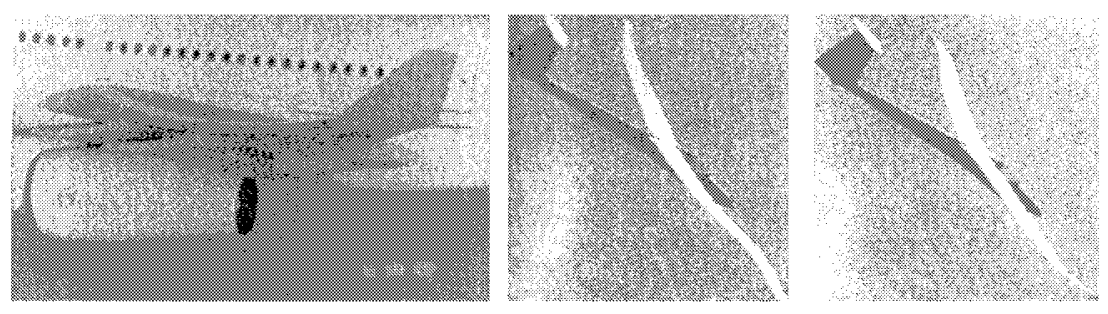

Figure 1. Deformation of a transport aircraft wing (I) and of a sailplane (r)

As long as strength requirements are fulfilled, structural flexibility itself is not necessarily objectionable. But the static and dynamic deformations of lifting surfaces generate steady and unsteady aerodynamic reactions, that in turn alter the deformations. These interactions may lead to several different aeroelastic problems with far-reaching technical consequences for flight. Of special importance is the flutter stability of wings and control surfaces. Particularly during the First World War pilots often reported of heavy oscillations of the wings and tail planes, that resulted in many fatal accidents. Back then aircraft were usually biplanes and the most frequent aeroelastic problems did occur not 
on the wings but with tail flutter. One of the first documented cases was the tail flutter problem of the British Handley-Page 0/400 bomber. Biplane constructions had a high torsional wing stiffness due to the interplane bracing. This was lost when cantilever monoplanes were developed, and flutter became even more dangerous. A remarkable example is the destruction of a big Ju90 aircraft in 1938 by bending-torsional tail-plane flutter. Although these accidents were not recognized to be a stability problem at that time, it seemed clear from the beginning that a theoretical investigation of these destructive vibrations required knowledge of both the elastic oscillatory behaviour of the lifting surfaces and the oscillatory motion-induced unsteady airloads. This presented a great scientific challenge. A systematic investigation of wing flutter and unsteady aerodynamics began about 1920 in Goettingen, Germany and in Turin, Italy. In general three different forces interact and generate aeroelastic effects, namely elastic forces $E$, aerodynamic forces $A$ and inertial or mass forces $I$. They are distributed continuously over an aircraft and are in equilibrium :

$$
E(q)+A(q)+I(q)=Q
$$

$Q$ denotes a known external force, which is independent from the aeroelastic system, rising for example from: atmospheric turbulence, gusts, landing gear impacts. $E, A, I$ are functions of the elastic geometrical deformations $q$. If $Q=0$, the equation describes the general aeroelastic stability problem. The analytical solution of this problem thus requires the calculation of the involved system forces as a function of local elastic deformations for a complete aircraft or for its components and the solution of the equilibrium equation.

The governing equations of fluid dynamics, the Navier-Stokes equations, have been known since the 19th century. But with the exception of a few limiting special cases there was no chance of finding analytical solutions. Only the development of numerical CFD (Computational Fluid Dynamics) methods since 1970 opened the way to computing particularly compressible and viscous flow problems around complex $3 \mathrm{D}$ configurations. When powered flight began, mainly experimental observations were used together with simplifications of the basic equations in order to derive mathematical models of the flow around an aircraft. In $1918 \mathrm{~L}$. Prandtl developed his idea of a lifting line to represent the effect of a lift generating wing. Since the mathematical value of the circulation is directly related to the lift, the wing is replaced by a vortex line along the span at $1 / 4$ chord of the wing, with constant strength $\Gamma$. Flow visualisations first in water tunnels for $2 \mathrm{D}$ wing sections for a starting motion showed the presence of a bound vortex and of a free starting vortex of opposite sign, which is swept down from the trailing edge to infinity by the main flow. These two vortices compensate, thus fulfilling the vorticity conservation law. If the fluid is incompressible and free of rotation - except for the bound and free vortices - 
a potential function exists for the velocity vector and fulfills the Laplace equation. This follows from mass conservation, while a relation between pressure and velocity follows directly from momentum equation:

$$
\operatorname{rot} \vec{v}=0 \rightarrow \vec{v}=\nabla \phi \text { and } \frac{\partial \rho}{\partial t}+\nabla(\rho \vec{v})=0 \quad \rho=\text { const } \rightarrow \nabla \vec{v}=\Delta \phi=0
$$

In 1922 Prandtl extended his concept to a theory of unsteady oscillating 2D lifting surfaces. Every time the bound vortex strength $\gamma$ - and thus the lift changes, a small free vortex with the opposite strength $\epsilon$ of this change is created to fulfill the vorticity conservation law, and is carried downstream from the trailing edge by the main flow. This model of unsteady free and bound vortices was applied by Prandtl's student W. Birnbaum, who developed a mathematical singularity method for computing unsteady airloads induced by an oscillating 2D wing [17]. He showed that aerodynamic lift and moment on the oscillating surface lag behind the forcing motion, and that this phase shift as well as the magnitude of unsteady lift and moment strongly depend on a new similarity parameter, the reduced frequency, see fig. 2 .

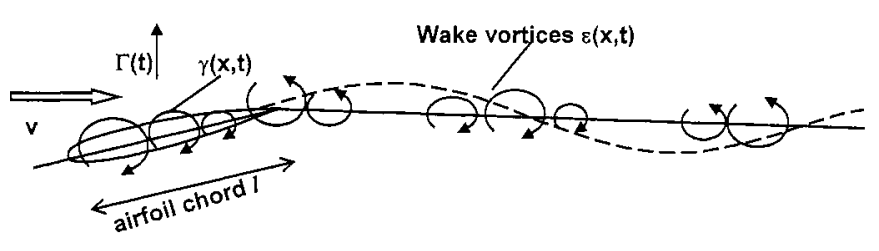

Figure 2. Vortex generation in the wake of an oscillating airfoil

He further remarked that modeling the unsteady problem requires a chordwise vorticity distribution instead of one bound vortex. His work was key to understanding the physical mechanism of flutter as a dynamic aeroelastic stability problem. This could be solved by assuming all forces including the unsteady aerodynamics to be harmonic and then treating eq.(1) as a complex Eigenvalue problem. Birnbaum died in 1925 at the age of only 28 .

Structural dynamic modeling was either provided by a simplified elastic beam theory or from experimental ground vibration tests. These soon reached a high standard. In contrast the prediction of unsteady airloads remained a key problem, due to the mathematical complexity. Measurements of unsteady motioninduced aerodynamics did not exist before 1938, and therefore the flutter test, either in a windtunnel or in flight, provided the only -indirect - validation of unsteady aerodynamic models. 
Birnbaum's theory was later expanded by Kuessner and Schwarz in Germany to include also control surface oscillations. Derivations of other solutions for the same problem were performed in Italy by Possio's colleague Cicala and in the US by Theodorsen, adopting mathematical singularity models or conformal mapping. As their results agreed, the development of unsteady incompressible $2 \mathrm{D}$ flow around airfoils was completed about 1930. The efforts that followed focussed on 3D and compressible flow.

When flight speeds increased, new problems were encountered, especially when sonic speeds were approached. A significant amount of flight stability was lost, control surfaces lost their efficiency or even reversed their effect and wings and rudders started heavy vibrations. The only chance to understand these phenomena was to investigate the influence of the compressibility on unsteady flows. The special complexity of compressible flow was already known at the beginning of the last century. If flow velocity $v$ was no longer much smaller than the speed of sound $a$, the speed of disturbance propagation in the fluid began to play an essential role, and the governing velocity potential equation for unsteady flow became a wave equation, see fig. 3
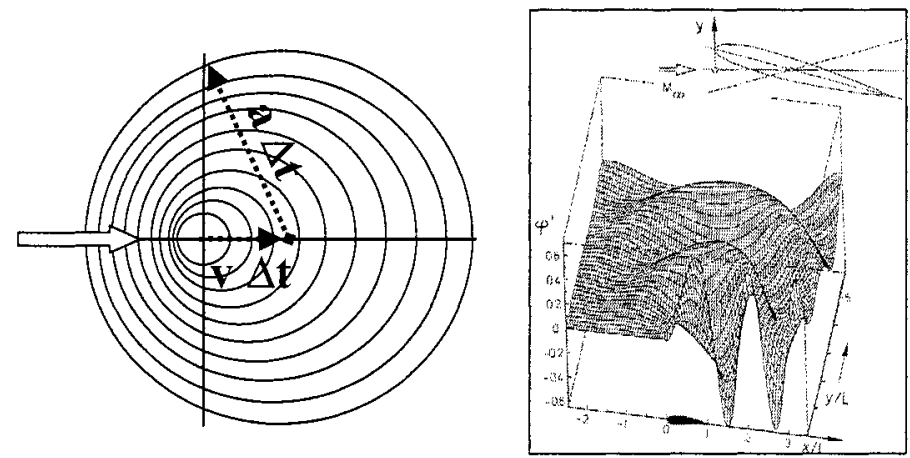

Figure 3. Disturbance wavefronts in a compressible flow (1) and spatial waviness of the velocity potential value ( $r$ )

The priciples of compressible flow, including supersonic flows and shock waves, had already been elaborated around 1900 as gasdynamic theories. The only practical application of this knowledge was in the design and analysis of steam turbines and in the research of ballistic projectiles. Propeller blade tips had already reached these velocities and after 1930 several airplanes reached velocities higher than $600 \mathrm{~km} / \mathrm{h}$, among them the German Messerschmidt 109 and 
the first jet airplane He178VI and the Italian waterplane MC72. For practical aircraft applications Prandtl and Glauert derived that lift and pressure on wings in compressible flow depend on Mach number by the factor $\beta=\sqrt{1-M a^{2}}$. Mach number $M a=v / a$ denotes the ratio between fight velocity and isentropic speed of sound. A major milestone on the path of compressible flow research was the 5th Volta Congress of the Royal Italian Academy of Sciences in 1935 in Rome. This conference with the topic of "High Velocities in Aviation" had been initiated by Arturo Crocco, a general and aeronautical engineer. The most famous aeronautical scientists of that time were invited to present state of the art in high-speed flow research. Several topics adressed at this conference soon influenced the work of young scientists like Camillo Possio in the following years.

\section{First publications until 1937}

In [1] and [2] Camillo Possio investigated supersonic flow problems. The choice of this topic was certainly influenced by the Volta Congress. In supersonic flow about bodies of revolution, like ballistic projectiles, often a curved shock wave occurs, which abruptly decelerates the flow from supersonic to subsonic and produces a strong drag on the body. Behind non-curved shocks the flow around a prescribed sharp nosed geometry could already be calculated by the method of characteristics. For the case of a body with blunt nose and a curved shock the flow becomes nonisentropic and rotational downstream of the shock. This law was named after L. Crocco, the son of A. Crocco. The method of characteristics is no longer valid, but $\mathrm{L}$. Crocco outlined a method to overcome this dilemma at the Volta Congress, and he demonstrated that a mathematical solution could be obtained by a method presented by C. Ferrari, one of Possio's teachers. Camillo Possio expanded Crocco's work by deriving a concept for general 3D flows, like inclined bodies of revolution or wings.

Possio's key paper [2] on unsteady supersonic flow about airfoils remained unknown in the international scientific community for several years. This might be due to the fact that it was published in the "Pontificia Accademia Scientiarium Acta", and that its summary was written in Latin. Possio started his theory by deriving the velocity potential of an acoustic source of pulsating strength that moves rectilinear with supersonic velocity. This method was later also adopted by Kuessner for his General Lifting Surface Theory. Possio derived the induced velocity component normal to the airfoil which was represented by a flat plate. He combined the effects of two different sources, with an infinitesimal small distance to the upper and lower side of the thin plate, and thus obtained the effect of a doublet singularity with pulsating strength. The whole unsteady 2D supersonic flow was then modeled by a doublet distribution along the chord of the plate, and the doublet strength distribution was calculated 
from the normal velocity which in turn was forced by the airfoil oscillation, the so-called downwash $w$.

\section{Possio's integral equation}

In 193824-year old Camillo Possio outlined the very first theory for unsteady compressible flow around 2D oscillating airfoils [3]. This was a complicated problem, because for the unsteady compressible wave equation the use of vortices as elementary solutions was not possible and the Prandtl-Glauert rule did not hold for unsteady flow. The wave equation for the velocity potential is derived from mass- and momentum conservation, and by linearly superimposing steady and unsteady flow velocity components $\vec{v}=u_{\infty}+\nabla \varphi \rightarrow \phi=\varphi+u_{\infty} x$ the following form is obtained:

$$
\left(1-M a^{2}\right) \frac{\partial^{2} \varphi}{\partial x^{2}}+\frac{\partial^{2} \varphi}{\partial y^{2}}-2 \frac{M a^{2}}{v} \frac{\partial^{2} \varphi}{\partial x \partial t}-\frac{M a^{2}}{v^{2}} \frac{\partial^{2} \varphi}{\partial t^{2}}=0
$$

As for isentropic flow the density is a function of pressure only, the acceleration vector has a potential $\psi$ too, which is a function of pressure only and thus has no discontinuity in the flow except at compression shocks and at the lifting surface, but not in the wake. Prandtl had presented the concept of the acceleration potential at the Volta Congress:

$$
\rho \frac{D v}{D t}=-\nabla p \rightarrow \frac{D v}{D t}=-\nabla \int \frac{d p}{\rho}=\nabla \psi
$$

Adopting a Galilei-Transformation $x=\bar{x}-v t, y=\bar{y}, t=\bar{t}$ Possio derived a corresponding wave equation and fundamental solution $\psi_{D}(x, y)$ in form of a doublet singularity of strength $A(\xi, \eta)$.

$$
\begin{gathered}
\frac{\partial^{2} \psi}{\partial \bar{x}^{2}}+\frac{\partial^{2} \psi}{\partial \bar{y}^{2}}-\frac{1}{a^{2}} \frac{\partial^{2} \psi}{\partial \bar{t}^{2}}=0 \\
\psi_{D}=A v^{2} \frac{4 i}{\beta} e^{i\left(\omega t+\frac{\omega^{*} M a(x-\xi)}{\beta^{2} l / 2}\right)} \frac{\partial}{\partial \eta} H_{0}^{(2)}\left(\frac{\omega^{*} M a}{\beta^{2}} \frac{\sqrt{(x-\xi)^{2}+\beta^{2}(y-\eta)^{2}}}{l / 2}\right)
\end{gathered}
$$

The reduced frequncy is defined by $\omega^{*}=\omega b / v$ and $b=l / 2$ is half chord. If the oscillating flat plate is modeled by doublet distribution of varying strength along the chord, the total acceleration potential is computed by an integral with a singularity at $(x, y)=(\xi, \eta)$. Evaluation of Cauchy's mean value, yields a relation between doublet strength and pressure jump across the plate, and the doublet strength can be calculated from the boundary condition, which prescribes the disturbance velocity $w$ in $y$-direction induced by the airfoil oscillation. Performing differentiation and the limiting process $y \rightarrow 0$ and $\eta \rightarrow 0$ 
finally yields Possio's equation

$$
\begin{aligned}
\bar{w}=w & (x, t) e^{-i \omega t}=\frac{\omega}{\rho v^{2}} \int_{-l / 2}^{+l / 2} \delta p(\xi) K\left[M a, \omega^{*} \frac{(x-\xi)}{l / 2}\right] \\
K= & \frac{1}{4 \beta}\left\{e^{i M a \Xi}\left[i M a \frac{|x-\xi|}{x-\xi} H_{1}^{2}(\Xi)-H_{0}^{2}(\Xi)\right]\right\} \\
& +\frac{i \omega^{*}}{4 \beta} e^{-i M a \Xi} \int_{-\infty}^{x-\xi} d u H_{0}^{2}(\Xi) e^{i \frac{\omega^{*} u}{b \beta^{2}}}
\end{aligned}
$$

The kernel $\mathrm{K}$ depends on Mach number and reduced frequency and contains cylindrical Hankel functions. As Possio did not find an analytical expression for the kernel, he numerically calculated tables of $K$ for $\mathrm{Ma}=0,0.25,0.50$, 0.75 and for a limited range of values $\Xi=\omega^{*}|x-\xi| M a /\left(\beta^{2} b\right)$. Using these values he solved the integral equation by a series expansion for the unknown pressure jump $\delta p$ according to Birnbaum and Ackermann

$$
\delta p(\theta)=A_{0} \cot \frac{\theta}{2}+\sum_{n=1}^{\infty} A_{n} \sin n \theta \cos \theta=\xi / l
$$

Possio applied only 3 terms of this series and correspondingly solved his integral equations by fulfilling it for $w$ at 3 chorwise positions. This approximation was sufficient for rigid heave and pitch motions, and the unsteady components of lift and moment coefficients read

$$
c_{L}=\frac{L}{\rho l v^{2}}=\frac{\pi}{2}\left[A_{0}+\frac{1}{2} A_{1}\right] \quad c_{M}=\frac{M}{\rho l^{2} v^{2}}=\frac{\pi}{16}\left[A_{1}+\frac{1}{2} A_{2}\right]
$$

These results are valid for sinusoidal oscillations and depend linearly on the oscillation amplitude and on its time derivative around the mean position. Possio presented his results in a form that was common in Italy and the UK, see fig. 4. The paper was translated in Germany in 1939, and the diagrams were transferred to the German standard definition of coefficients, which differed from Possio's. This work supported engineers in Germany with unsteady compressible airloads in a form directly applicable for flutter computations. For more complex oscillation modes, like trailing edge flaps, which encounter a discontinuous behaviour at the hinge axis, more terms would have to be calculated. In 1942 Dietze suggested an iterative scheme that is based on the development of the kernel function in terms of its simpler incompressible counterpart, which had been presented by Possio in his paper too.

The outstanding character of Possio's key paper was mentioned soon by Kuessner [18] in 1940: "The oscillating wing for $0 \leq M a \leq 1$ has been investigated 

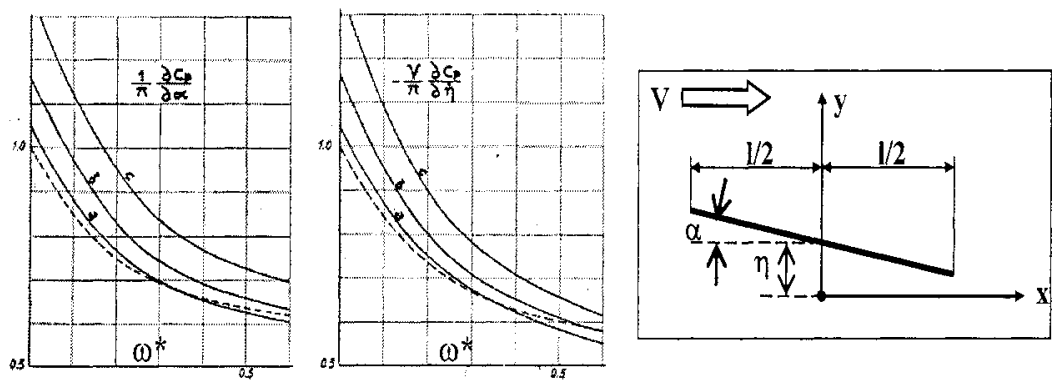

Figure 4. Results from Possio's original paper

up to now in just a single investigation for the two dimensional problem" and in 1981 by Garrick and Reed [23]: "There followed shortly afterward two short outstanding contributions by Camillo Possio in Italy. In 1938 he applied the acceleration potential to the two-dimensional nonstationary problem and arrived at an integral equation (Possio equation)".

\section{Further unsteady aerodynamic contributions}

With increasing flight speed $v$, the aerodynamic loads acting on an aircraft structure increase like $v^{2}$. Thus high elastic bending moments appeared for wings with large aspect ratio (ratio of wing span to chord). The need to investigate wings with low ratios was also driven by the requirements of more agile military aircraft and by the role of tail planes in flutter. As a result the effects of the wing tips became important and unsteady 3D theories had to be developed. In $3 \mathrm{D}$ flow the bound vortex line on the wing, together with the free vortices behind the tip and the starting vortex far downstream form a closed vortex line. For 3D unsteady flows around an oscillating wing the 2D unsteady model of Birnbaum and the 3D steady model of Prandtl were refined by Sears, see fig. 5

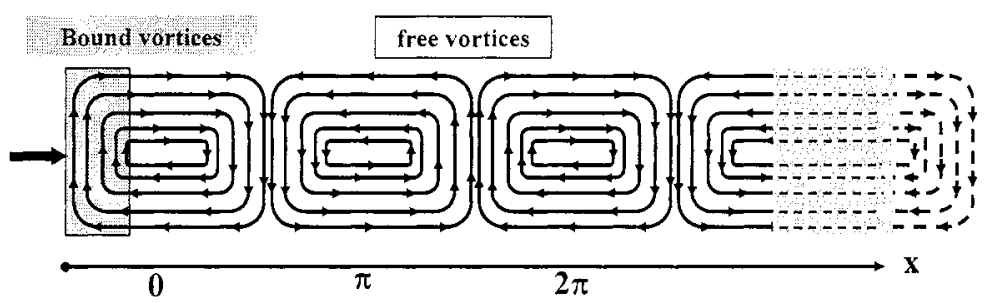

Figure 5. Lines of constant vorticity in the wake of a 3D oscillating wing 
Vortex rings change periodically in strength and sign and leave the trailing edge of the oscillating wing, which forms the unsteady wake. The figure reflects that spanwise vorticity strength varies, thus producing a system of free vortex lines. Calculation of the interaction between the system of free vortices and bound vortices was too difficult whereas the simple model of just one single bound vortex was too coarse. Different authors - v.Borbely in Germany, Sears in the UK, Jones in the USA, and Cicala and Possio $[8,9,11,12]$ in Italy developed different methods to approximate the varying vortex strengths for both bounded and free vortices. In 1943 Kattenbach in Germany performed a comparison of the different theories for a $3 \mathrm{D}$ wing of elliptical planform in heavy oscillations with different reduced frequencies in incompressible flow. Remarkably he spent much more effort in reviewing Possio's theory in his paper than the other ones. After the war unsteady incompressible 3D problems were solved as a special case of Kuessners General Lifting Surface Theory, as soon as computers became available.

Several high-speed wind tunnels, several of them in Germany and in Guidonia near Rome, as well as both flutter and unsteady aerodynamic tests in high speed flow were planned in the 1930s, but tests were not realized before the end of the war. One of the problems with test results is that the model is tested in an airstream, which is bounded either by the free atmosphere or by the walls of the test section. Camillo Possio was among the first ones to investigate the influence of tunnel walls on oscillating models [15]. He first demonstated, that tunnel wall disturbances decreased with frequency like $1 / \omega$. Then he calculated the effects of the above mentioned closed walls and free jet conditions. He found that esp. the imaginary part of unsteady lift coefficient was significantly changed by the wall effects. In [6], [7] and [8] Possio investigated the problem of general unsteady motions in $2 \mathrm{D}$ and $3 \mathrm{D}$ flows, namely non-harmonic impulsively started motions.

\section{Influence on the development of 3D theories}

In Germany H.G Kuesnner in 1940 published his General Lifting Surface Theory, which was the first German paper dealing with unsteady compresssible flow. The strategy he chose for derivation of his theory, use of a Lorentz Transformation to derive a wave equation for the velocity potential of a moving source, was the same that Possio had used two years earlier. Kuessner was very familiar with Italian work on aerodynamics and aeroelasticity, because he reviewed several translated papers, including 10 by Possio. Kuessner derived an integral equation relating the unknown load distribution on a lifting surface $\delta p(\xi, \eta)$ and the prescribed downwash velocity amplitude $\bar{w}(x, y, z)$ normal to 
the surface, by means of a new Kernel function $K$.

$$
\bar{w}=\iint d \xi d \eta \frac{\delta p}{4 \pi \rho v} e^{i \omega \frac{\xi-x}{v}} \frac{\partial^{2}}{\partial z^{2}} \int_{-\infty}^{x-\xi} d \lambda \frac{e^{i \omega\left(\lambda-M a \sqrt{\lambda^{2}+\beta^{2}(y-\eta)^{2}+\beta^{2} z^{2}}\right)}}{\sqrt{\lambda^{2}+\beta^{2}(y-\eta)^{2}+\beta^{2} z^{2}}}
$$

The theory is valid for lifting thin surfaces of an arbitrary planform in inviscid and irrotational flow, but not in transonic flow. Kuessner demonstrated that all other known theories of that time were special cases of this theory, but the Kernel was left in the form of a highly singular integral, whose solution could only be found for special cases like Possio's 2D theory. Since no analytical solution was found before the war, Possio's theory of 2D strips along the span of wings and tail planes was applied for the flutter analysis in high-speed flight.

\section{Work in non-aerodynamic fields}

The topic of lateral firing from an airplane was probably chosen in 1939 due to Camillo Possio's military service. The spinning axis and path of a projectile fired from board of an aircraft in a direction different from the aircraft's flight direction are not parallel. This yields an effective aerodynamic incidence angle, and therefore aerodynamic side forces. Possio calculated the airloads and the projectile motion and showed that due to the spinning forces the axis of the projectile soon turns to the direction of the path [10]. In 1941 Possio focused on hydrodynamic problems. He investigated the influence of the free water surface on moving underwater airfoils [13], [14]. He calculated forces on a $2 \mathrm{D}$ hydrofoil, the motion of which is governed by the Laplace equation for the velocity potential. The effect of the free water surface is assumed to be a linear perturbation. Possio used the constraints, that disturbance velocity vanishes at infinity and that pressure on the water surface has a constant value. Modeling both the hydrofoil and the disturbance velocity by a vortex distributions Possio derived the result that lift and drag on the hydrofoil depend on the Froude number $F r=v / \sqrt{g l}$ and on the depth in which the hydrofoil of chord length $l$ moves with velocity $v, g$ denotes the gravitational constant.

\section{Extension of Possio's method after the war}

Before the war 2D compressible unsteady flow based on Possio's integral equation was the best method available to model unsteady airloads in flutter calculations. 3D unsteady methods existed only for incompressible flow, and it was not before the 1960s that 3D unsteady compressible fiow could be calculated by solving Kuessners equation. The Doublet Lattice Method (DLM)[19] became the standard method for the years that followed. Similar to Possio's 2D theory, the flow is modeled by a doublet distribution on the lifting surfaces. The unknown doublet strength is discretized on trapezoidal panels. This powerful 
method vields large linear systems of equations and fully populated matrices

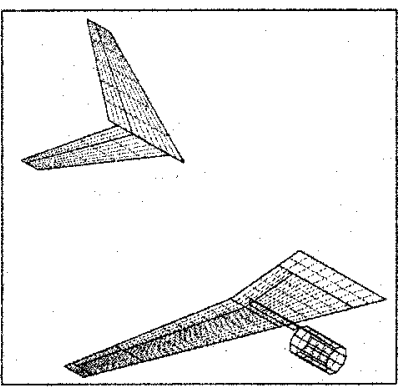

Figure 6. Discretization of an aircraft in DLM (1) and in TDLM (r)

A transonic extension, the so-called TDLM (Transonic Doublet Lattice Method) has been developed later on [20]. In this method the unsteady transonic flow is modeled as a perturbation of a mean transonic steady flow and is governed by a nonhomogeneous wave equation for the acceleration potential. In contrast to purely subsonic flow additional source singularities have to be distributed in the flow field, the strength of which depends on the velocity potential of the transonic steady flow. Thus the DLM model of doublet panels is extended by volume elements of constant source strength, see fig. 6 .

$$
\frac{\partial^{2} \psi}{\partial x^{2}}+\frac{\partial^{2} \psi}{\partial y^{2}}+\frac{\partial^{2} \psi}{\partial z^{2}}+\lambda^{2} \psi=\left(\frac{\partial}{\partial x}+i \frac{\omega^{*}}{\beta^{2}}\right) \sigma\left[\frac{(\gamma+1) M a^{2}}{\beta^{2}} \frac{\partial \phi^{0}}{\partial x}\right]
$$

Flutter became a problem in turbomachines when attempts to increase efficiency and to reduce noise and emissions made the size of aircraft engines and thus the blade size grow. Additional geometrical parameters as well as blade-to-blade interactions play a significant role. A method developed in 1973 Carstens [21] extended Possio's integral equation to the case of a 2D cascade blade row, which is a model of a surface cut through a fan at constant radius. The kernel of the integral equation depends not only on Mach number and reduced frequency but also on stagger angle $\lambda$, interblade distance $\tau$ and interblade phase angle $\theta$. In [21] efficient numerical methods are developed both for this kernel and for the original Possio kernel. The computational results show the interesting effect of blade resonance. For constant values of the Mach number and the reduced frequency the lift coefficient of a reference blade varies strongly when the interblade phase angle is changed, and even drops to zero for specific resonance values. This effect is typical for unsteady compressible flows, and appears when 
disturbance waves propagating in a compressible fluid are reflected between boundaries within a time period fitting the oscillation period of the boundary motion. This effect was also observed in wind tunnel tests on oscillating models. Fromme and Golberg [22] reformulated Possio's kernel for a 2D airfoil in order to include the boundary conditioins of wind tunnel walls and were able to compute the effects of wind tunnel wall resonance.

These examples are demonstrating, that fast analytical methods are still of high value, if the influence of several parameters in unsteady aerodynamics has to be scanned. The performance of similar studies with modern CFD methods requires a tremendous effort.

\section{Conclusion}

Comparing today's capabilities of unsteady aerodynamic computation with those of 60 years ago, much progress has been made due to the CFD development and the enormous growth of computer power. The first unsteady nonlinear transonic flow simulations with complex shock motions were obtained around 1975. They were based on nonlinear potential equations, and were soon followed by solutions of the inviscid Euler- and the viscous Reynolds Averaged Navier-Stokes (RANS) equations. All of these methods adopt finite volume or finite difference methods to model the conservation laws of mass momentum and energy together with gas equations of state and different turbulence models. Today they allow for computations of 3D flows around complete aircraft configurations with strong shock waves and flow separation in the whole speed range. Flutter computations are beginning to be performed by directly integrating the equations of motion of the structure in time, together with computing the interaction between structural deformations and fluid dynamics. Such flutter simulations usually take many hours of CPU, while calculations of the flutter boundary as an Eigenvalue problem with Possio's aerodynamics needs just seconds or just minutes if TDLM aerodynamics is chosen.

We can conclude that Possio's legacy is manifold. First his mathematical models for unsteady aerodynamics are still valuable and his papers are still being cited. Secondly he paved the way for Kuessner's theory and for later 3D unsteady compressible methods like DLM and TDLM in addition to sophisticated analytical methods in turbomachinery. Third he has shown, how fruitful analytical methods can be for understanding of physical mechanisms and for providing engineers with a feeling for the importance and magnitude of different parameters, both aspects of which are sometimes neglected today.

\section{References}

[1] C. Possio. Sul moto razionale dei gas. Atti Accad. Naz. Lincei, Rend. VI. S. 25, 455-461, 1937 
[2] C. Possio. L'azione aerodinamica sul profile oscillante alle velocita ultrasonore. Pontificia Accademia Scientiarium Acta 1, 93-106, 1937

[3] C. Possio. L'azione aerodinamica sul profilo oscillante in un fluido compressibile a velocita iposonora. L'Aerotecnica 18, 441-459, 1938

[4] C. Possio. L'azione aerodinamica su una superficie portante in moto oscillatorio. Atti Accad. Naz. Lincei, Rend. VI. 28, 194-200, 1938

[5] C. Possio. Determinazione dell'azione aerodinamica corrispondente alle piccolo oscillazioni del velivolo. L'Aerotecnica 18, 1323-1351, 1938

[6] C. Possio. Sul moto non stazionario di una superficie portante.Atti Accad. Sci. Torino 74, 285-299, 1939

[7] C. Possio. Sol moto non stazionario di un fluido compressibile Atti Accad. naz. Lincei Rend. VI, 29, 481-487, 1939

[8] C. Possio. L'azione aerodinamica su di una superficie portante in moto vario Atti Accad. Sci. Torino 74, 537-557, 1939

[9] C. Possio. Sulla determinazione dei coefficienti aerodinamici che interessano la stabilita del velivolo Comm. Pontif. Acad. Sci.). 3, 141-169, 1939

[10] C. Possio. Sullo sparo di fianco da bordo di un aereo. Atti Accad. Sci. Torino 74, 1939

[11] C. Possio. Sul problema del moto discontinuo di un ala. Nota 1. L'Aerotecnica 20,655681,1940

[12] C. Possio. Sul problema del moto discontinuo di un ala. Nota 2. L'Aerotecnica 21, 205 230,1941

[13] C. Possio. Sulla teoria del moto stazionario di un fluido pesante con superficie libera. Ann. Mat. pura appl. IV, 20, 313-329, 1941

[14] C. Possio. Campo di velocita creato da un vortice in un fluido pesante a superficie libera in moto uniforme. Atti Accad. Sci. Torino 76, 365-388, 1941

[15] C. Possio. L'interferenza della galleria aerodinamica nel caso di moto non stazionario. $L$ ' Aerotecnica 1940-XVIII. 1940

[16] C. Possio. The influence of the viscosity and thermal conductibility on sound propagation. Atti Accad. Scienze Torino 78. 274-292. 1943

[17] W. Birnbaum. Das ebene Problem des schlagenden Flügels. ZAMM 4. 277-292. 1924

[18] H.G. Kuessner. Allgemeine Tragflächentheorie. LuFo 17. 370-378. 1940

[19] E. Albano, W. Rodden. A doublet lattice method for calculating lift distributions on oscillating surfaces in subsonic flow. AIAA Journal 1969-7. 279-285. 1969

[20] S. Lu, R. Voss. TDLM - a Transonic Doublet Lattice Method for 3D potential unsteady transonic flow calculation and its application to transonic flutter prediction. Proc. IFASD 1993 77-95. AAAF 1993

[21] V. Carstens. Berechnung der instationaeren Druckverteilung an harmonisch schwingenden Gittern in ebener Unterschallstroemung. DFVLR-IB 73-J06 and 75-J02. 1973 and 1975

[22] J.A. Fromme, M.A. Golberg. Aerodynamic interference effects on oscillating airfoils with controls in ventilated windtunnels. AIAA Journal 18.417-426. 1980

[23] I.E. Garrick, W.H. Reed III. Historical development of flutter. Journal of Aircraft 18. 897-912. 1981 